\title{
Correspondence
}

\section{Nuclear mitotic apparatus protein, NuMA, modulates p53-mediated transcription in cancer cells}

\author{
A Endo ${ }^{1,3}$, A Moyori $^{1,3}$, A Kobayashi ${ }^{1}$ and RW Wong ${ }^{\star, 1,2}$ \\ Cell Death and Disease (2013) 4, e713; doi:10.1038/cddis.2013.239; published online 4 July 2013 \\ Subject Category: Cancer
}

\section{Dear Editor,}

The nuclear mitotic apparatus protein (NuMA) is mainly distributed inside the nucleus during interphase. During mitosis, NuMA functions in focusing microtubules toward the mitotic spindle poles and bundling spindle microtubules to centrosomes. NuMA is a $237-\mathrm{kD}$ a protein with the discontinuous coiled-coil spacer between $\mathrm{N}$ - and C-terminal globular domains that can form parallel coiled-coil dimers $\approx 200 \mathrm{~nm}$ in length. ${ }^{1}$ We previously reported that NuMA interacted with RNA export factor 1 (Rae1) during mitosis ${ }^{2}$ and this interaction is important for spindle bi-polarity formation. ${ }^{3,4}$ On the other hand, the role of NuMA in interphase cells is understood to a lesser extent. Here, we first examined whether transient overexpressed NuMA altered cell cycle progression. Human NuMA (full-length) tagged with GFP at the N-terminus was expressed in HeLa cells (Supplementary Figure 1a left panel). We next determined whether cell cycle progression would be altered by NuMA overexpression by FACS analysis, we found that overexpressed NuMA led to the slight reduction in apoptotic cells fraction (indicated as *) from $8.6 \%$ to $5.3 \%$ ( $n=3$ sets of independent experiments; Supplementary Figure 1a right panel).

Interestingly, we and others found that nuclear pore proteins ( $\mathrm{Tpr}^{5}$ and $\mathrm{Nup}^{6} \mathrm{8}^{6}$ ) associated with tumour suppressor p53, causing growth arrest, apoptosis and autophagy, through the regulation of a different set of target genes. ${ }^{5,6}$ p53 is the most commonly mutated gene in human cancer and is frequently referred to as the 'guardian of the genome'. In response to genomic stresses, p53 and p21 drive cell cycle arrest giving more time for genomic damage to be repaired before cell division or moves to apoptosis eliminating irreparably damaged cells. ${ }^{7}$ As Rae1 is also associated with Nup98, it led us to surmise the potential regulatory mechanism between NuMA and p53.

To further address this possibility, we tested the mRNA and protein expression of several p53-target genes by quantitative RT-PCR (qPCR), immunoblotting and confocal microscopy. In those transfecting with GFP-NuMA in HeLa cells, we found upregulation of p53 mRNA (Supplementary Figure 1b upper panel). Besides, we observed the enhanced mRNA expression of p53 downstream genes p21 (cell arrest) and PUMA (apoptosis) but not DRAM (autophagy) in triplicate experiments (Supplementary Figure 1b upper panel). Consistently with qPCR data, we found ectopic overexpressed GFP-NuMA markedly increased of p53 and p21 protein expression, while only faint signals in GFP-vector alone control cells via confocal microscopy (Supplementary Figure 1c) and immunoblotting experiments (Supplementary Figure $1 \mathrm{~b}$ lower panel). Our results reveal for the first time that ectopic expression of NuMA can manipulate endogenous p53 and p21 transcriptional expression during interphase. In this regard, it will be particularly interesting to examine the detail mechanism in apoptosis via the upregulation of NuMA-p53 interaction in near future.

During this study, a report came online also showing NuMA is required for the selective induction of p53-target genes, including p21. ${ }^{8}$ Consistent with their findings, our preliminary data also find that NuMA siRNA did not alter the nuclear morphology, gross chromatin structure, though we cannot exclude the possibility that NuMA partial depletion might change the microenvironments of nuclear compartments or our current NuMA depletion protocol might be insufficient to discover the visible changes of nuclear morphologies (data not shown). More importantly, forthcoming studies should be designated to elucidate the detailed mechanisms of how NuMA regulated p53 in mammalian cell cycle, as neither NuMA nor p53 have been identified in yeast.

\section{Conflict of Interest}

The authors declare no conflict of interest.

Acknowledgements. This work was supported by Grants from MEXT Japan and the Asahi Glass Foundation, the Suzuken Memorial Foundation, the Sumitomo Foundation, the Kowa life science Foundation, the Mochida Memorial Foundation, the Sagawa Foundation, the Uehara Memorial Foundation, the Inamori Foundation and the Takeda Science Foundation (to RW).

1. Radulescu AE, Cleveland DW. Trends Cell Biol 2010; 20: 214-222.

2. Wong RW, Blobel G, Coutavas E. Proc Natl Acad Sci USA 2006; 103: 19783-19787.

3. Nakano H, Wang W, Hashizume C, Funasaka T, Sato H, Wong RW. Cell Cycle 2011; 10: $425-433$.

\footnotetext{
${ }^{1}$ Laboratory of Molecular and Cellular Biology, Department of Biology, Faculty of Natural Systems, Institute of Science and Engineering, Kanazawa University, Kakuma-machi, Kanazawa 920-1192, Japan and ${ }^{2}$ Bio-AFM Frontier Research Center, Kanazawa University, Kakuma-machi, Kanazawa, Japan ${ }^{*}$ Corresponding author: RW Wong, Laboratory of Molecular and Cellular Biology, Department of Biology, Faculty of Natural System, Institute of Science and Engineering, Kanazawa University, Kakuma-machi, Kanazawa 920-1192, Japan. Tel: + 8176264 6250; Fax: + 8176264 6253; E-mail: rwong @ staff.kanazawa-u.ac.jp

${ }^{3}$ These authors contributed equally to this work.
} 
4. Bukata L, Parker SL, D’Angelo MA. Curr Opin Cell Biol 2013; doi:10.1016/i.ceb.2013.03.002.

5. Funasaka T, Tsuka E, Wong RW. Sci Rep 2012; 2: 878.

6. Singer S, Zhao R, Barsotti AM, Ouwehand A, Fazollahi M, Coutavas E et al. Mol Cell 2012; 48: $799-810$

7. Vogelstein B, Lane D, Levine AJ. Nature 2000; 408: 307-310.

8. Ohata H, Miyazaki M, Otomo R, Matsushima-Hibiya Y, Otsubo C, Nagase T et al. Mol Cell Biol 2013; 33: 2447-2457.
Cell Death and Disease is an open-access journal published by Nature Publishing Group. This work is licensed under a Creative Commons Attribution-NonCommercialNoDerivs 3.0 Unported License. To view a copy of this license, visit http://creativecommons.org/licenses/by-nc-nd/3.0/

Supplementary Information accompanies this paper on Cell Death and Disease website (http://www.nature.com/cddis) 